\title{
Structures and Dynamics of Solar Filaments - challenges in observing and modeling
}

\author{
Oddbjørn Engvold \\ Institute of Theoretical Astrophysics \\ University of Oslo \\ PO Box 1029 Blindern \\ N-0315 Oslo, Norway \\ email: oddbjorn.engvold@astro.uio.no
}

\begin{abstract}
Fundamental information about the nature of solar filaments and governing physical processes are retained in their small-scale structure and dynamics. The paper reviews some recent high resolution studies of filaments, with emphasize on potential impact on current understanding of their physical nature.
\end{abstract}

\section{Introduction}

Solar filaments which are seen in absorption against the solar disk are the central cool core of the enigmatic filament channels. They provide, indirectly, information about the magnetic fields in these regions at sub-arc sec resolution. The term 'cool' means temperatures around $10^{4} \mathrm{~K}$ and is here relative to the more than 100 times hotter surrounding corona. When filaments are observed in emission beyond the solar limb they are referred to as prominences. This notation is also used below.

Filament channels are contained in magnetic arcades straddling regions of opposite polarities in the photosphere underneath. Coronal cavities are frequently observed between prominences and these overlying arcades (cf Tandberg-Hanssen, 1995). The cavity regions are detectable basically by their lack of emission (Serio et al., 1978; Wiik, Schmieder and Noëns, 1993). Schmieder et al. (2004) combined EUV observations of a tall, polar crown filament, obtained with SOHO's EIT and SUMER instruments, with TRACE and highresolution $\mathrm{H} \alpha$ filtergrams from La Palma, in a study of its overall temperature structure. Viewing the filament partly from one side, the authors notice the presence of a diffuse, dark extension in EUV, below the $\mathrm{H} \alpha$ filament, which is interpreted as a coronal 'volume blocking' by a cool plasma. Further investigations with similar data combinations, may tell how this EUV extension is related to cavities in filament channels.

Direct measurements of magnetic fields in solar prominences yield field strengths in the range from 3 to 20 Gauss (Leroy, 1988; Bommier et al., 1994), which is sufficient for the magnetic field to control the plasma. Indirect methods offer possibilities to study the magnetic field topology at scales $\leqslant \frac{1}{2}$ arc second. The shapes and dynamics of the low- $\beta$ filament plasma reflect a fragmented topology of these fields. The Balmer $\mathrm{H} \alpha$ line is widely used for such studies of filaments at their smallest scales since its optical density here is usually about unity and since sub-arc sec resolution is attainable with ground-based telescopes at these wavelengths.

Given that the 3-D character of solar filaments is not readily interpreted from 2-D filtergram data, the question regarding how magnetic fields in filaments connect with the surrounding corona and photosphere, therefore cannot be easily answered. By combining motions of individual features in the plane of the sky and in the line of sight one may derive the true velocity vector of the motion, and hence also the direction of the magnetic 


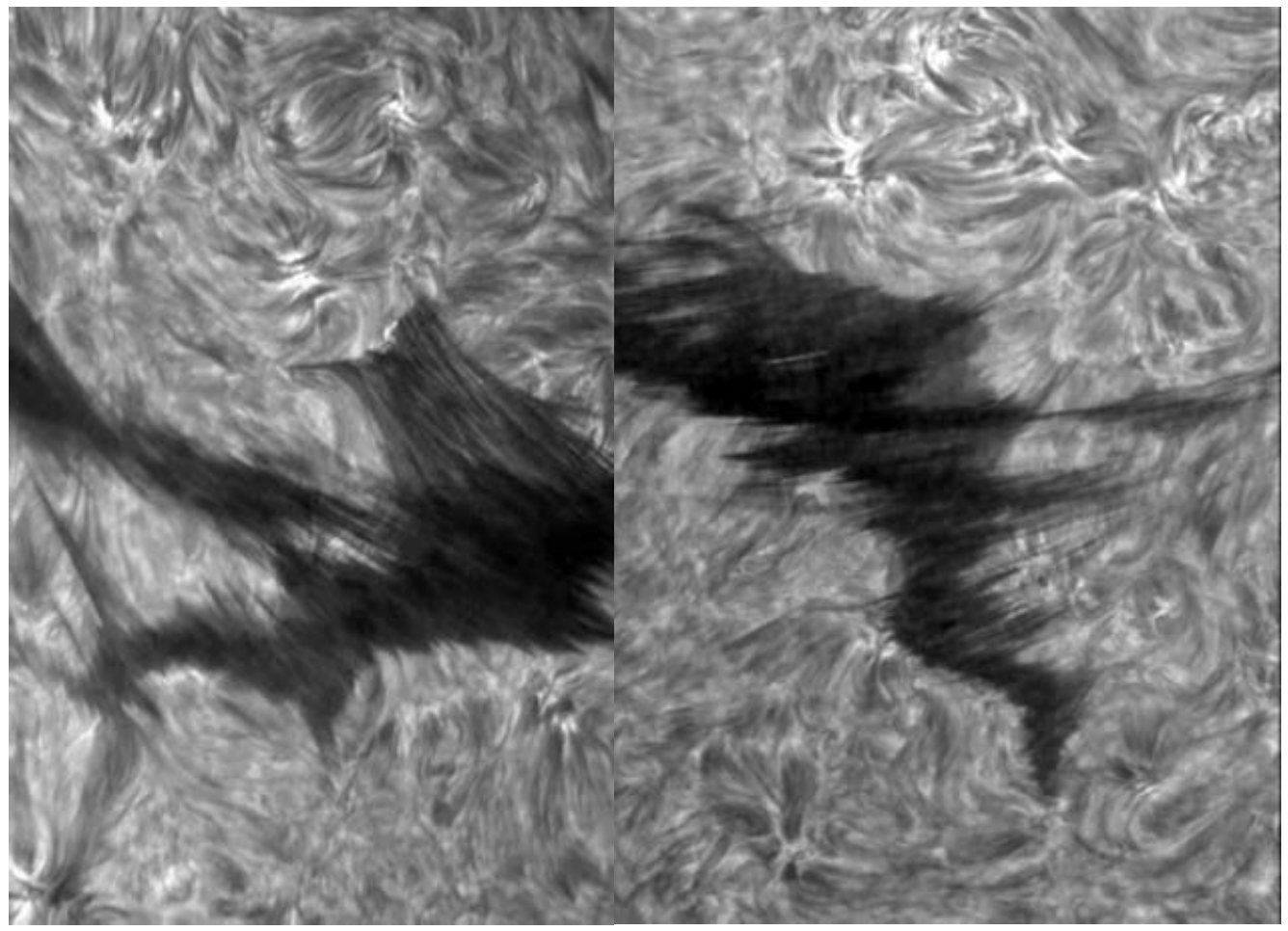

Figure 1. The two H $\alpha$ filtergrams showing parts of a quiescent filament observed on 25 and 26 August, 2003, respectively, with the new Swedish solar telescope. The images are part of time series observed with the narrow-band LMSAL/SOUP filter.

fields. This technique has been applied by Lin et al. (2003) to a large polar crown filament and it appears promising.

Filament barbs are occasionally seen to connect with observable magnetic fields in the solar photosphere (Martin, 1998). However, a major fraction of photospheric fields that take part in the formation and existence of the cool filament plasma, could be too weak to be detected with current observational techniques. Canceling of magnetic fields which is assumed to be a prime mechanism leading to filament formation could therefore go un-noticed.

We shall here review briefly some new and ongoing studies of small-scale dynamics of solar filaments, with the aim to illustrate current challenges in observations as well as in modeling of solar filaments. It is not the intention to present a comprehensive picture of past and ongoing research in this field. Important issues concerning formation of filament channels are not touched here.

\section{Filament Structure and Dynamics}

\subsection{Threads}

The thread-like, small-scale structure of solar prominences have been recognized since they were first observed more than 130 years ago (see Livingston et al., 1987). Richard Dunn's movies of prominences observed in H $\alpha$ (Dunn, 1961; Menzel and Wolbach, 1960) have been widely used and appreciated by many. Figure 1 shows two recent examples of quiescent filaments observed with the new Swedish Solar Telescope at La Palma, using the narrow-band SOUP filter of Lockheed-Martin Solar and Astrophysics Laboratory. These 

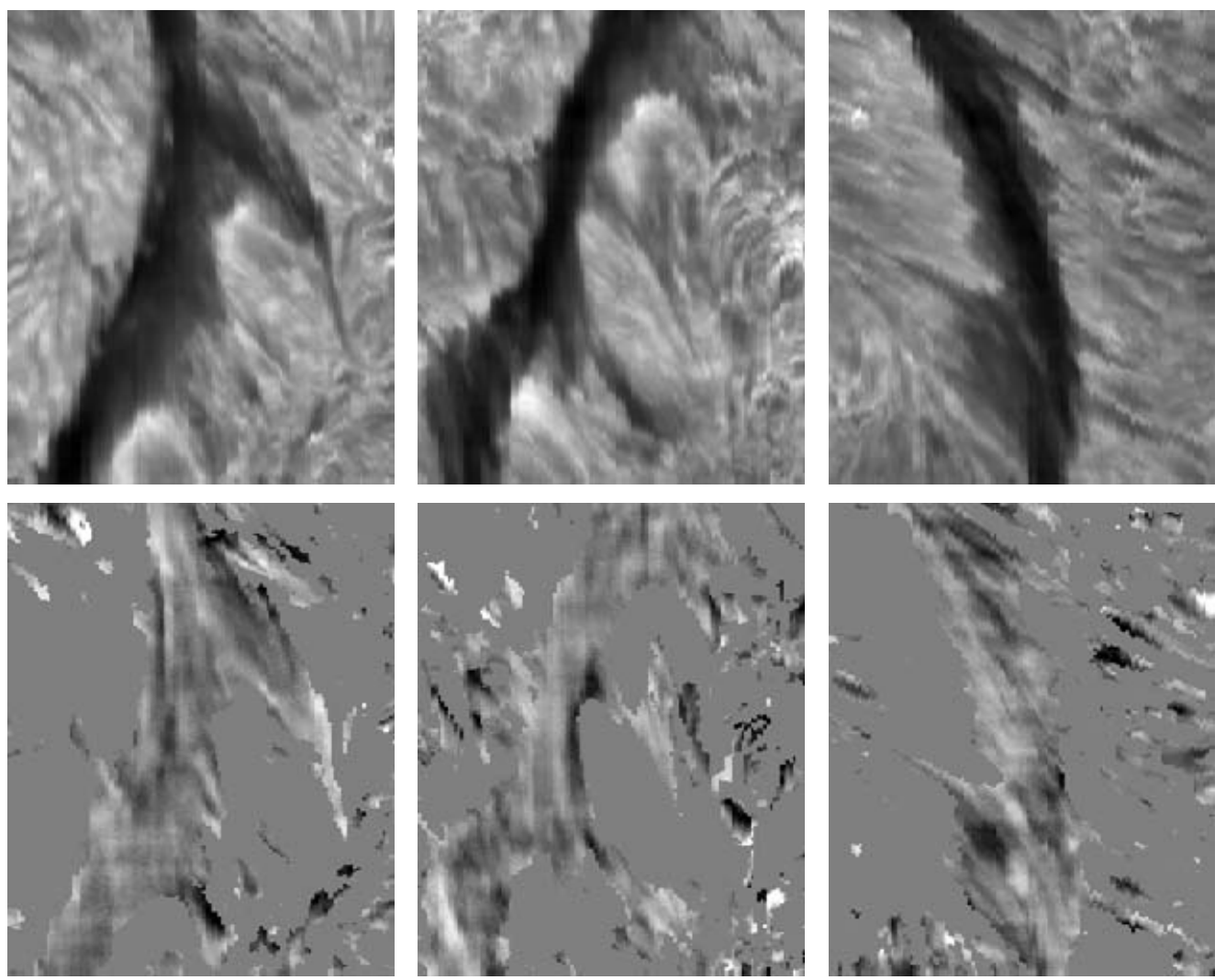

Figure 2. The three upper panels show different filaments located along the same filament channel in the northern atmosphere 6 May 2000. The monochromatic $\mathrm{H} \alpha$ images were obtained from spectral frames recorded as the solar image was stepped across the spectrographic slit. The image scale is $60 \times 90$ arc second $^{2}$. The lower panels show corresponding Doppler velocities, where dark and bright signals represents, respectively, red and blue shifts. In areas where the line intensity is higher than the intensity of the filaments are the Doppler signals set equal to zero in order to outline the location of the filaments (Wiik et al., 2005).

images demonstrate the ubiquity of very fine, long threads. Lin et al. (2004a) report that the widths (FWHM) of these threads are $\leqslant 0.3$ arc sec. The fact that the width of the thinnest of the threads is comparable to the diffraction limit of the telescope of about 0.15 arc sec, at the wavelength of $\mathrm{H} \alpha$, suggest that even thinner threads may exist.

From the same data Lin (2004) finds that individual threads move sideways and shift position at speed up to $3 \mathrm{~km} \mathrm{~s}^{-1}$. This is in agreement with line-of-sight velocity fluctuations in a large prominence observed by Zirker and Koutchmy $(1990 ; 1991)$. Such relative motion of individual threads is probably a consequence of their linking to the dynamic photosphere.

Filament threads are thought to be thin magnetic flux tubes filled with absorbing cool plasma. Lin et al. (2004a) find that the visible lengths of the threads vary from a few arc sec up to as much as 25 arc sec. Threads change with time by fading and plasma flowing. The amount of cool material within a given thread will be constrained in the event that it is the result of condensation of hot material in the channel.

Observations of thin filament threads close to telescope resolution, require very stable conditions in order to discriminate between true changes in the absorption and seeing. 

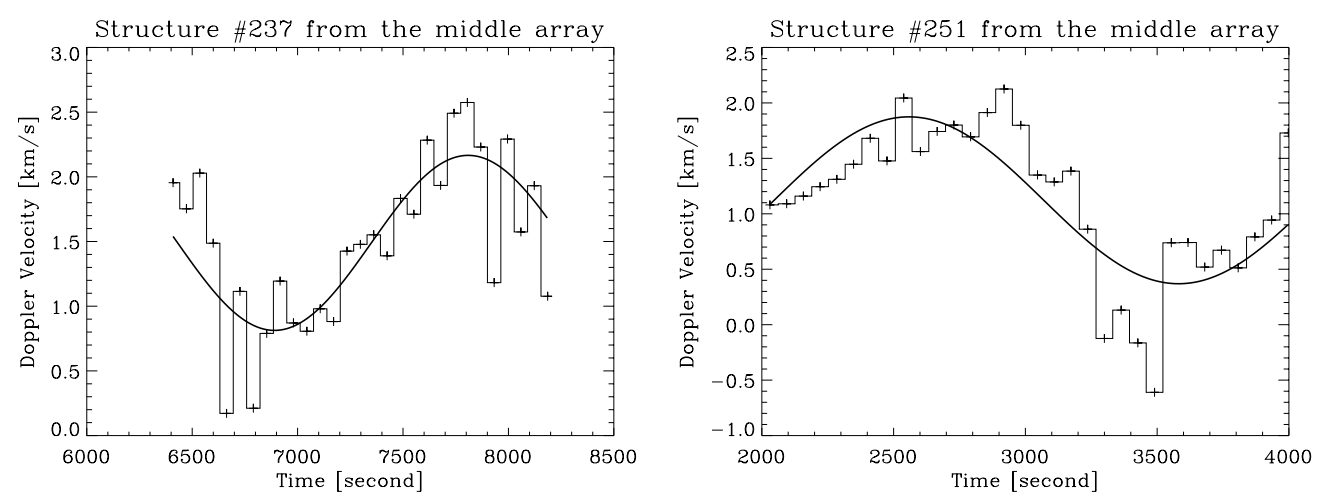

Figure 3. The diagrams show the variation of the line-of-sight velocity of two moving features and their approximated sine curves. The left panel yields a mean velocity $V_{l o s} \simeq 1.5 \mathrm{~km} \mathrm{~s}^{-1}$. The corresponding transverse velocity, $V_{t r}$, is $-4.8 \mathrm{~km} \mathrm{~s}^{-1}$, which gives a net velocity $\mathrm{V}=-5.0$ $\mathrm{km} \mathrm{s}^{-1}$. For the right hand panel one gets $V_{l o s} \simeq 1.1 \mathrm{~km} \mathrm{~s}^{-1}, V_{t r} \simeq-3.8 \mathrm{~km} \mathrm{~s}^{-1}$ and $V=-4.0$ $\mathrm{km} \mathrm{s}^{-1}$ (see Lin et al. 2003). (The notations used here are, $t r=$ transverse motion, i.e. in the plane of the sky, and los = motion in the line of sight.)

\subsection{Flows and oscillations}

The streaming (and counterstreaming) of filament plasma have been demonstrated by Zirker et al. (1998) and Lin et al. (2003). From more recent observations Lin et al. (2004b) find flow velocities along individual threads corresponding to $15 \pm 10 \mathrm{~km} \mathrm{~s}^{-1}$, which therefore is taken to be field-aligned flowing of the plasma. Higher flow velocities are observed in 'activated' prominences with TRACE (Kucera, Tovar \& De Pontieu, 2003).

Again, one sees flowing in opposite directions in threads located closely apart. Obviously, one cannot decide uniquely from 2-D images how such threads with oppositely directed flows are located relative to each other in space. The counterstreaming character of flows is also illustrated in Figure 2 obtained from Wiik et al. (2005). One sees here both outward and inward directed flows in barbs.

Figure 3 from Lin et al. (2003) shows the variation of line-of-sight velocities of two filament structures as they move along with the flows. The diagrams illustrate the well known oscillatory nature of solar filaments and prominences (Yi and Engvold, 1991; Thompson and Schmieder, 1991; Balthasar et al. 1993; Schutgens, 1998). Large filaments are known to oscillate, largely in phase, over their entire bodies with periods slightly less than 30 minutes to an hour, while individual threads oscillate with periods of some minutes (see Engvold, 2001). The character of the oscillations reflects the magnetic field strength and topology (cf Oliver \& Ballester, 2002) and how these fields are rooted in the photosphere below.

\subsection{Thermal structure}

Recent studies based on EUV data address the issue of the thermal structure within and surrounding the cool filament plasma. Cirglinao et al. (2004) derived Differential Emission Measure in a large prominence, based on emission lines covering a temperature range from $\leqslant 310^{4} \mathrm{~K}$ to more than $310^{5} \mathrm{~K}$, and the authors conclude that there is at least 40 times more emitting material at the highest temperature compared with that of the plasma seen in $\mathrm{H} \alpha$. Figure 4 shows the thermal cross section of a thread with transition region implying a hot cavity region. In the event that filament channels also should contains thin, cool material, as implied by studies of Schmieder et al. (2004), 

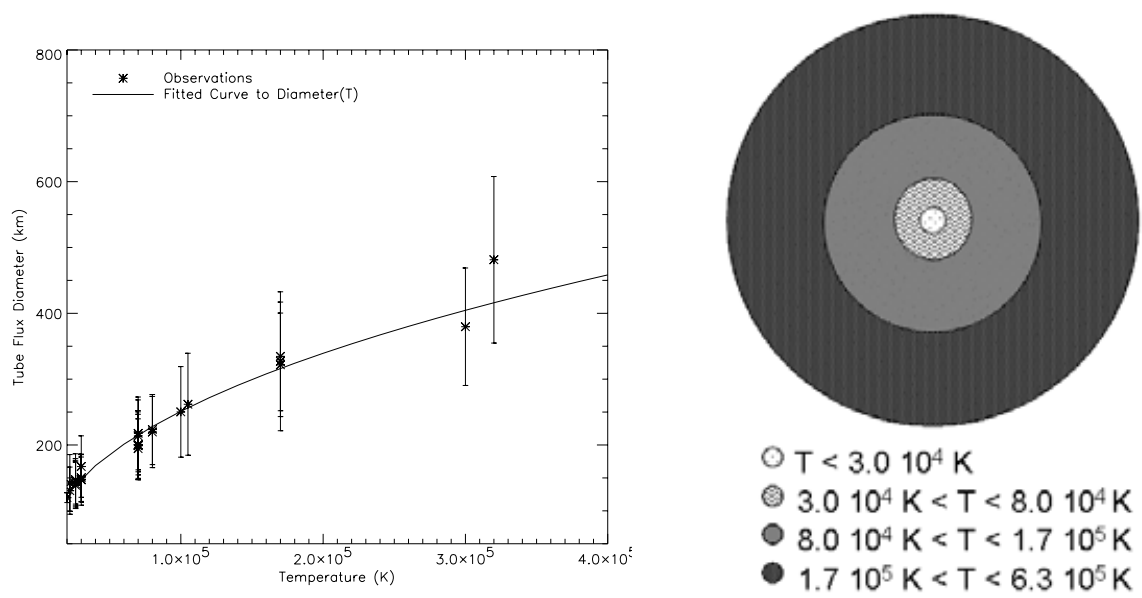

Figure 4. Differential Emission Measure studies of the Prominence-Corona Transition Region yield 40 times more emitting volume at coronal temperature as compared to about $20,000 \mathrm{~K}$. The figure illustrates the temperature profiles in the case that the threads are surrounded by a transition region. (From Cirglinao et al. (2004).)

these must be separated from the hot cavities and evidently located below the cool $\mathrm{H} \alpha$ filaments.

\subsection{How do filaments connect with the photosphere?}

The feet of the characteristic arches of prominences are located at supergranulation (SG) cell boundaries, according to Rompolt \& Bogdan (1986) and Forbes \& Malherbe (1986). Lin et al. (2004a) have recorded time series of filaments in $\mathrm{H} \alpha$ simultaneously with white light images from the photosphere immediately below. The objective was to study the orientation of filaments with respect to SG boundaries, using the well known technique of Local Correlation Tracking (November \& Simon, 1988). Figure 5 from Lin et al. (2004a) shows two filament images with corresponding SG cell boundaries superimposed. In the left image one sees that endpoints of filament barbs favor SG cell boundaries. Several SG cells are seen to cross the Polarity Inversion Line. In the image to the right the picture is rather different. One of the barbs appears there to be pointing into a SG cell. Other cases that have been studied from the same data set, give results similar to the second example. One concludes from this that some barbs point to SG cell boundaries whereas the general picture is that barbs most often connect with regions inside SG cells where the kGauss fields are less prevalent.

For one case in data obtained with the Swedish Solar Telescope, Lin (2004) finds that individual threads seem to be rooted in dark inter-granular lanes. This is not surprising given that magnetic network flux is usually observed in intergranular lanes. So-called Bright Points in G-band filtergrams correspond to magnetic elements. At locations where threads appear to be rooted, however, one finds no discernible Bright Points, which may suggest that the magnetic fields there is weaker than the general network field.

It is highly desirable to be able to detect and measure weak magnetic fields in the photosphere with sub-arc second resolution, since such fields also are potential precursors for filament formation. 


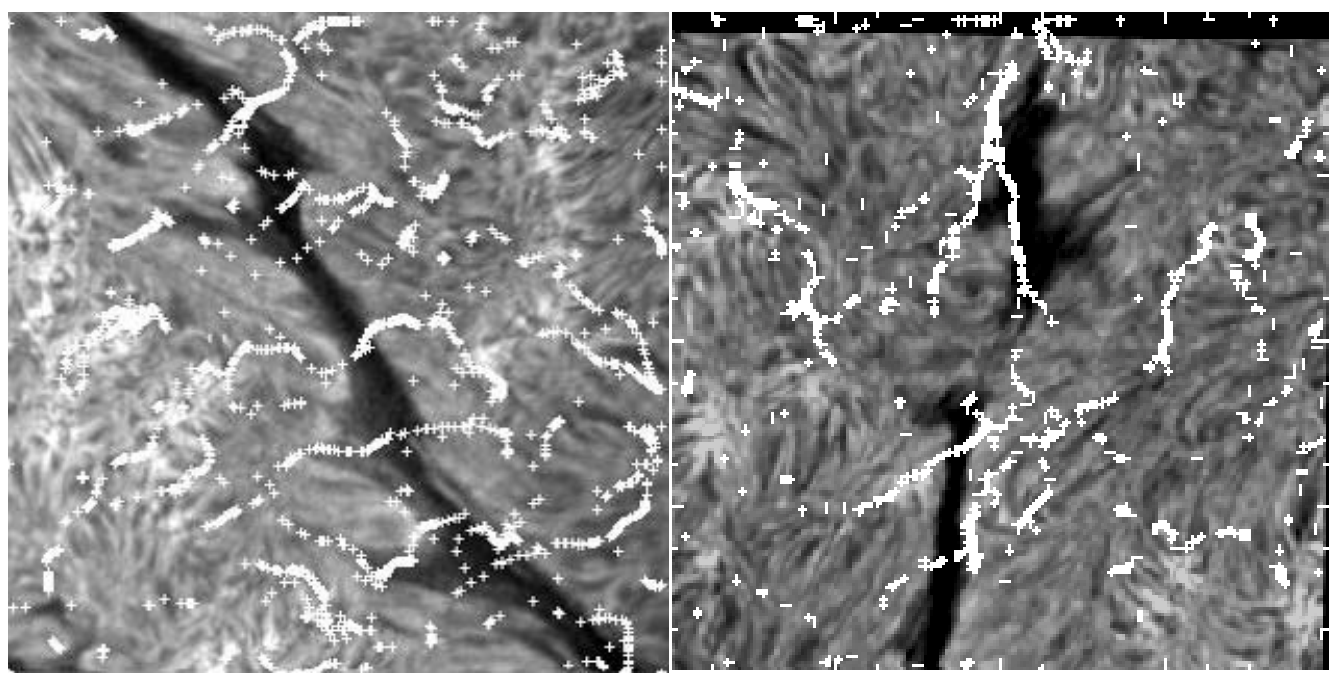

Figure 5. The two images illustrate how filaments observed in $\mathrm{H} \alpha$ are located on top of supergranulation cells, from a study by Lin et al. (2004a). The distribution of supergranulation cells are derived by application of the LCT technique.

\section{Concluding remarks}

Key issues in current understanding of formation and existence of solar filaments (and prominences) are, such as, the origin of the cool matter high above the photosphere, and how the cool plasma is supported in regions of inclined magnetic fields. From this follows questions concerning how and where filaments are rooted in the photosphere below, and the role of magnetic field cancellation and/or pressure induced inflow of mass may lead to formation of filaments. Furthermore, what is causing filaments to oscillate and the ever present flowing of the plasma? Also, further high-resolution, multi-wavelength investigations shall be encouraged (Stellmacher \& Wiehr, 2000).

The suitability of static approximations in modeling of solar filaments must be carefully tested and discussed in light of what observations reveal about their variability and dynamics. It is conceivable that the mechanism that causes the plasma to flow at speeds around $15 \mathrm{~km} \mathrm{~s}^{-1}$, and higher, also may provide support against gravity in barbs where the magnetic fields presumably are inclined. Acceleration by waves should be further pursued, theoretically (Pecseli \& Engvold, 2002).

\section{Acknowledgements}

The author wishes to acknowledge stimulating collaborations with Yong Lin, Sara Martin, Brigitte Schmieder, Einar Tandberg-Hanssen, Luc Rouppe van der Voort, Jun Elin Wiik and Jack Zirker in his studies of solar prominences and filaments. Travel related to discussions with US collaborators was in part supported by NASA Grant NAG5-4180 and NAG5-10852 to Helio Research.

\section{References}

Bommier, V., Landi Degl'Inocenti, E., Leroy, J.-L., \& Sahal-Brechot, S. 1994, Solar Phys. 154, 231

Cirglinao, K., Vial, J.-C., \& Rovira, M. 2004, Solar Phys. 223, 94

Balthasar, H, Wiehr, E., Schleicher, H., and Wöhl, H. 1993, Astron. Astrophys. 277, 635

Dunn, R.B. 1960, Ph.D. Thesis Harvard University 
Engvold, O. 2001, in INTAS Workshop on MHD Waves in Astrophysical Plasmas Universitat de les Illes Balears, eds.: J.L. Ballester \& B. Roberts, p.123

Forbes, T. \& Malherbe, J.M. 1986, Astrophys. J. Letters 302, L67

Kucera, T.A., Tovar, M. \& De Pontieu, B. 2003, Solar Phys. 212, 81

Leroy, J.-L. 1988, in Solar and stellar coronal structure and dynamics. Proceedings of the ninth Sacramento Peak Summer Symposium, Sunspot National Solar Observatory, p. 422

Lin, Y. 2004, PhD Thesis, University of Oslo

Lin, Y., Wiik, J.E. \& Engvold, O. 2003, Solar Phys. 216, 109

Lin, Y., Engvold, O., Wiik, J.E., Rouppe van der Voort, L., \& Frank, Z.A. 2004a, Solar Phys. (Submitted)

Lin, Y., Engvold, O., Rouppe van der Voort, L., Wiik, J.E. \& Berger, T. 2004b, Solar Phys. (In press)

Livingston, W.C., Engvold, O. \& Jensen, E. 1987, Astronomy 15, 18

Martin, S.F. 1998, Solar Phys. 182, 107

Mein, P., Mein, N., \& Wiik, J.E. 1994, Solar Phys. 151, 75

Menzel, D.H. \& Wolbach, J.G. 1960, Sky and Telescope 20, 119

Oliver, R. \& Ballester, J.L. 2002, Solar Phys. 206, 45

Rompolt, B. \& Bogdan, T. 1986, in Coronal and Prominence Plasmas Ed. A.I. Poland. NASA Conf. Publ 2442, p.81

Pecseli, H. \& Engvold, O. 2000, Solar Phys. 194, 73

November, L.J. \& Simon, G.W. 1988, Astrophys. J. 333, 427

Schmieder, B., Lin, Y., Heinzel, P., \& Schwartz, P. 2004, Solar Phys. 221, 297

Schutgens, N. 1998, PhD Thesis, Utrecht University

Serio, S., Vaiaba, G.S., Godoli, G., Motta, S., Pirronello, V. \& Zappala, R.A. 1978, Solar Phys. 59,65

Stellmacher, G. \& Wiehr, E. 2000, Solar Phys. 196, 357

Tandberg-Hanssen, E. 1995, The Nature of Solar Prominences Kluwer Academic Publisher

Thompson, W.T. \& Schmieder, B. 1991, Astron. Astrophys. 243, 501

Wiik, J.E., Schmieder, B. Noëns J.C. 1993, Solar Phys. 149, 51

Wiik, J.E.,, Lin, Y., Engvold, O, Martin, S.F., \& Zirker, J.B. 2005, Solar Phys. (in preparation)

Yi, Z. \& Engvold, O. 1991, Solar Phys. 134, 275

Yi Z. 1992, PhD Thesis, University of Oslo

Zirker, J.B., Engvold, O., \& Martin, S.F. 1998, Nature 396, 440

Zirker, J.B. \& Koutchmy, S.. 1990, Solar Phys. 127, 109

Zirker, J.B. \& Koutchmy, S.. 1991, Solar Phys. 131, 107 


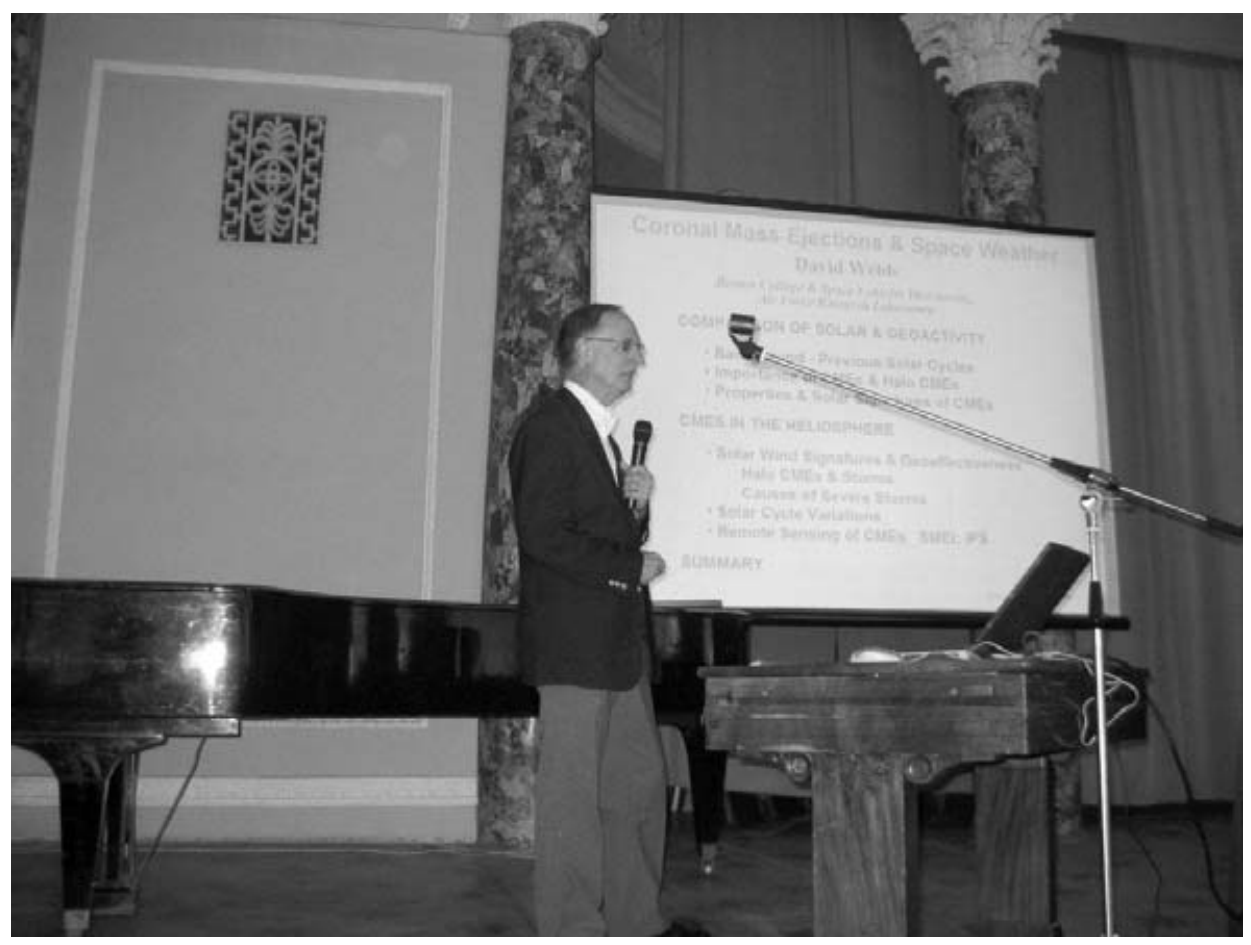

David Webb (USA).

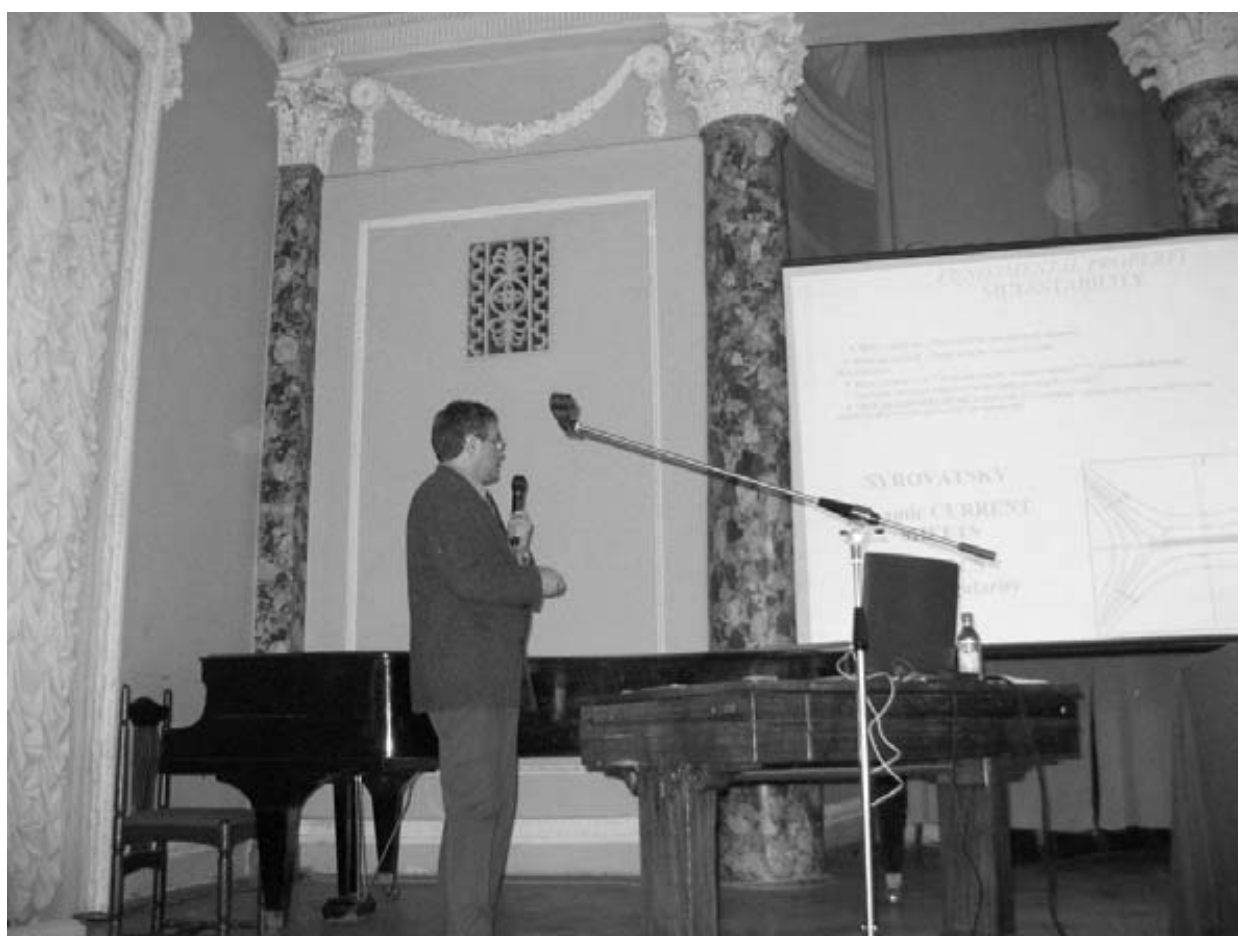

Lev Zelenyi (Russia). 